\title{
Os planos de recuperação econômica pós-Covid-19 na encruzilhada das mudanças climáticas
}

\author{
Post-Covid-19 Economic Recovery Plans \\ at the Crossroads of Climate Change
}

\author{
Luiz Enrique Vieira de Souza* \\ Marcelo Fetz ${ }^{* *}$ (iD
}

\section{RESUMO}

O presente artigo discute as implicações da crise econômica instaurada pela pandemia de Covid-19 sobre as políticas de mitigação das mudanças climáticas. Com base num levantamento bibliográfico a respeito das alterações na química atmosférica dos centros urbanos, apontamos que a queda nas emissões de gases responsáveis pelo efeito estufa representou uma consequência direta, porém não intencionada, das políticas de lockdown e distanciamento social. Dado que essa redução foi passageira e insuficiente para responder aos desafios da "emergência climática", apresentamos uma análise crítica sobre os pacotes de recuperação econômica pós-pandemia e o risco de que estratégias orientadas exclusivamente pela retomada do crescimento acentuem o "efeito rebote" com consequências irreversíveis e calamitosas para os esforços de mitigação das mudanças climáticas.

Palavras-chave: Covid-19, mudanças climáticas, crise econômica.

\footnotetext{
* Universidade Federal da Bahia, Salvador, BA, Brasil.

Doutor em Sociologia pela Universidade de São Paulo (2012). Professor do Departamento de Sociologia da Universidade Federal da Bahia.

lenriquesol@yahoo.com.br

${ }^{* *}$ Universidade Federal do Espírito Santos, Vitória, ES, Brasil.

Doutor em Sociologia pela Universidade Estadual de Campinas (2012). Professor do Departamento de Ciências Sociais da Universidade Federal do Espírito Santo.
} 


\begin{abstract}
This article discusses the implications of the economic crisis brought about by the Covid-19 pandemic to climate change policies. Based on literature review on alterations of the atmospheric chemistry in urban centers, we point out that the drop in greenhouse gas emissions represented a direct though unintended consequence of lockdown and social distancing policies. Considering that such reductions were temporary and insufficient to respond to the challenges of the "climate emergency", we present a critical analysis of post-pandemic economic recovery packages and the risk that strategies exclusively oriented towards the resumption of growth might accentuate the 'rebound effect' with irreversible and dire consequences for the efforts to mitigate climate change.
\end{abstract}

Keywords: Covid-19, climate change, economic crisis.

\title{
RESUMÉN
}

Este artículo investiga las consecuencias de la crisis económica provocada por la pandemia Covid-19 en las políticas para combatir el cambio climático. Con base en una revisión bibliográfica sobre los cambios en la química atmosférica en los centros urbanos, señalamos que la caída de las emisiones de gases responsables del efecto invernadero representa una consecuencia directa, pero no intencional, de las políticas de cierre y aislamiento social. Dado que esta reducción fue curta e insuficiente para responder a los desafíos de la "emergencia climática", presentamos un análisis crítico de los paquetes de recuperación económica pospandémica y de los riesgos de que las estrategias se orienten exclusivamente hacia la reanudación del crecimiento e intensifiquen el "efecto rebote" con consecuencias irreversibles y nefastas para los esfuerzos por contener el calentamiento global.

Palabras clave: Covid-19, cambio climático, crisis económica.

\section{Introdução}

No final de 2019, um novo tipo de coronavírus foi identificado na cidade de Wuhan, região central da província de Hubei na China (Zhu et al., 2020; Xiao \& Torok, 2020; Zhang \& Qian, 2020). A Organização Mundial da Saúde (OMS) foi notificada pelo governo chinês em 31 de dezembro e, em 7 de janeiro de 2020, o novo vírus foi denominado SARS-CoV-2. A Covid-19, como a doença ficou conhecida, produz uma série de sintomas, podendo levar a um quadro de crise respiratória aguda, além de outros problemas associados (Kucharski et al., 2020; Dolhnikoff et al., 2020). A OMS reconheceu a gravidade da crise e, no dia 12 de março de 2020, após constatada a rápida 
disseminação do vírus por vários países, classificou a doença como uma pandemia (Cuccinota \& Vanelli, 2020). Esse quadro global de saúde pública produziu reações sanitárias, especialmente em razão das semelhanças entre a Covid-19 e outras pandemias ocorridas ao longo da história, como a gripe espanhola em 1918, a varíola mexicana de 1967, a Sars em 2002, a gripe aviária em 2005 e a gripe suína de 2009 (Morens et al., 2020). Entre as principais ações para o achatamento da curva de contaminação, conforme recomendação de epidemiologistas, destacaram-se o distanciamento social e o fechamento total ou parcial das cidades, com a permissão de circulação somente de trabalhadores cujas atividades fossem consideradas essenciais pelas autoridades (Chinazzi et al., 2020).

A cidade de Wuhan, ground zero da pandemia, foi colocada em quarentena pelas autoridades sanitárias chinesas no dia 23 de janeiro de 2020. Ao final do mês, outras 15 cidades da província de Hubei decretaram algum tipo de restrição à circulação de pessoas (Li, Zhao \& Sun, 2020). Já o governo indiano impôs um lockdown nacional a partir de 24 de março (The Lancet, 2020). Em 8 de março, a Itália colocou a região da Lombardia em quarentena e, no dia seguinte, implementou políticas de restrição à circulação e aglomeração de pessoas em todo o território nacional (Chintalapudi et al., 2020). Outros países europeus seguiram o exemplo italiano, restringindo a movimentação de cidadãos e turistas nas semanas seguintes (Gilbert, 2020). Ao final do mês de março, com o aumento de casos nos EUA, a América do Norte tornouse o novo epicentro da doença, com restrição à entrada e circulação de pessoas (Ren, 2020). No Brasil, as cidades de São Paulo e Rio de Janeiro decretaram políticas de distanciamento social e regras mais estritas quanto à movimentação de pessoas a partir do mês de abril, que posteriormente também foram implementadas por outras cidades do país (Krecl et al., 2020; Siciliano et al., 2020; Dantas et al., 2020).

As políticas de distanciamento social e de fechamento total ou parcial das cidades produziram um pulso de decrescimento econômico em escala global, com impactos não intencionados sobre a química atmosférica e, mais especificamente, sobre o regime de emissões de gases e substâncias poluentes responsáveis pelas mudanças climáticas. Esse fenômeno reatualizou debates urgentes quanto ao futuro da humanidade, como Green New Deal, decrescimento, adaptação e mitigação às mudanças climáticas. Diante desse novo contexto, o presente artigo explora, por um lado, as contradições impostas pela pandemia de Covid-19 em relação aos seus efeitos sobre o meio 
ambiente e, por outro lado, a problemática absorção do debate sobre os pacotes de recuperação econômica por discursos políticos que apresentam a crise como passageira e insistem no "retorno à normalidade". Ao não reconsiderar criticamente o paradigma distópico-destrutivo em vigor nas sociedades industriais, a presente turbulência reitera padrões de outras crises vivenciadas ao longo do século 20, as quais foram repetidamente desperdiçadas, mesmo quando já se dispunha de conhecimento perito sobre as possíveis consequências catastróficas de médio e longo prazo para os ecossistemas.

Com base em publicações e relatórios científicos sobre as alterações na química atmosférica decorrente das políticas de lockdown e distanciamento social, a segunda seção explora o avanço da pandemia no mundo e seu efeito positivo sobre a emissão de gases responsáveis pelo efeito estufa e sobre a poluição do ar nas grandes cidades. Como essa redução de poluentes diz respeito a um fenômeno que se mostrou passageiro, a terceira seção apoia-se em documentos oficiais, manifestos político-científicos e artigos da imprensa internacional para discutir o teor das propostas de recuperação econômica, com foco em suas implicações contraditórias no que tange ao combate às mudanças climáticas. A partir de um diagnóstico de época, segundo o qual a pandemia de Covid-19 nos coloca diante de uma encruzilhada de futuros climáticos, a quarta seção apresenta uma discussão crítica a respeito dos sucessivos desperdícios históricos de crises semelhantes e estabelece uma comparação entre as modalidades contemporâneas do Green New Deal apresentadas por países do Atlântico Norte e a perspectiva teórica do “decrescimento". Por fim, lançamos uma interrogação para futuras agendas de pesquisa acerca da viabilidade de políticas efetivas de mitigação às mudanças climáticas num contexto também marcado pela corrida armamentista e por disputas geopolíticas entre as principais potências mundiais.

\section{Políticas de lockdown, mudanças na química atmosférica e "efeito rebote" nas emissões globais.}

É fato que as medidas de restrição total ou parcial à circulação, adotadas por governos locais como principal ação de enfrentamento à pandemia da COVID-19, provocaram a redução momentânea das emissões de gases do efeito estufa, bem como a diminuição da concentração de material particulado na atmosfera (Zambrano-Monserrate et al., 2020; McNeill, 2020; 
Wang et al., 2020). As políticas de restrição adotadas no Rio de Janeiro entre os dias 16 e 19 de março de 2020, por exemplo, reduziram em torno de $50 \%$ a circulação de veículos de transporte público, além de significativa diminuição do tráfego de veículos particulares. Com a adoção de medidas semelhantes na cidade de São Paulo, imagens de satélite constataram queda da presença de material particulado (Krecl et al., 2020; Siciliano et al., 2020; Dantas et al., 2020). Condições semelhantes foram igualmente verificadas na China, Itália, França, Índia, Espanha (Gilbert, 2020; Ren, 2020; Lal et al., 2020). Esse cenário de melhoras parciais chegou a ser visto como um possível turning point na condução da governança global das mudanças climáticas. No entanto, essa tendência foi revertida nos últimos meses de 2020, quando alguns países superaram o nível de emissões pré-pandemia (Harvey, 2020; Rosenbloom \& Markard, 2020). O potencial danoso desse "efeito rebote" tem chamado a atenção da comunidade científica, especialmente por conta de políticas no setor energético daqueles países que apresentam forte dependência em relação ao uso de combustíveis fósseis (Gerima, 2020).

Com efeito, dados registrados no início da pandemia indicaram um alívio momentâneo dos indicadores de qualidade atmosférica. De acordo com Li e colaboradores (2020), observaram-se reduções de 16-26\% para SO2 e 2947\% para NOx durante as duas primeiras fases de contenção do vírus na região do Delta do Yangtzé. Chen, Wang e Huang (2020) verificaram que, durante a quarentena na cidade de Wuhan, houve uma queda de 22,8 $\mu \mathrm{g} /$ m3 nas emissões de NO2 (na China a queda foi de 12,9 $\mu \mathrm{g} / \mathrm{m} 3$ ). Em grande parte da Índia, registraram-se quedas de 10\% para CO e 18\% para NO, enquanto $\mathrm{O} 3$ e SO apresentaram um aumento de 17\% durante o período de lockdown (Shresta et al., 2020). Kerimray e seus colaboradores (2020) destacam que, no Cazaquistão, as concentrações de CO e NO2 sofreram redução de 49\% e 35\%, respectivamente, enquanto os níveis de O3 tiveram um acréscimo de 15\%, se comparados aos 17 dias anteriores ao lockdown. Benzeno e tolueno apresentaram concentração de 2 a 3 vezes superiores às observadas entre 2015-2019.

Fenômenos semelhantes foram observados na Europa e nas Américas. A concentração de NO2 decresceu nas cidades de Madri (-33,3\% em fevereiro e $-47,5 \%$ em março), Paris $(-33,1 \%$ em fevereiro e $-5,6 \%$ em março), Oslo (-28,3\% em fevereiro e $-39,7 \%$ em março) e Viena $(-18,1 \%$ em fevereiro e -4,4\% em março). A cidade de Nova Iorque também demonstrou redução nas taxas diárias de emissão desse poluente. A concentração de 
O3 apresentou comportamento instável entre as cidades, com decréscimo em Madri e crescimento em Londres e Toronto. O mesmo ocorreu com a concentração diária de SO2 após o lockdown, sendo observada queda em Madrid e Vienna e aumento em Londres. Já a concentração de CO, nos meses de fevereiro e março, apresentaram dados inconstantes, com aumentos e reduções observados ao redor do mundo: em fevereiro, queda nas cidades de Amsterdam (-35,1\%), Londres (-48,3\%), Lima $(-27,3 \%)$ e aumento nas cidades de Bogotá $(+15,8 \%)$ e San Francisco (+22,6\%); no mês de março a queda foi mais consistente nas cidades de Lima, Londres, Nova York, São Francisco e Toronto.

A melhora nos indicadores de qualidade do ar foi igualmente observada no Brasil. De acordo com Nakada e Urban (2020), as concentrações de NO, NO2 e CO na cidade de São Paulo apresentaram redução de 77,3\%, 54,3\% e $64,8 \%$, respectivamente, se comparadas à média dos cinco anos anteriores. A concentração de ozônio, porém, apresentou aumento de 30\% no mês de março, provavelmente pela queda na concentração de dióxido de nitrogênio na atmosfera (Nakada \& Urban, 2020). Ao analisarem as emissões na cidade de São Paulo entre os meses de março e abril-período de início das recomendações de restrição adotadas pelo governo local - Krecl et al. (2020) verificaram diminuições significativas das concentrações dos óxidos de nitrogênio (NO e NO2). Na cidade do Rio de Janeiro, Siciliano, Dantas e Silva (2020) constataram redução nas concentrações de CO e NO2. Em contrapartida, a presença de ozônio aumentou em todas as localidades monitoradas ao longo do estudo. A redução nas emissões de gases poluentes, especialmente daqueles responsáveis pelas mudanças climáticas, pode ser sintetizada no gráfico 1. Esse fenômeno se deve aos efeitos do lockdown sobre determinadas atividades econômicas, em especial nos setores industrial e de transportes.

Já os gráficos 2 e 3 apontam no sentido de um efeito rebote verificado nos últimos meses de 2020. A retomada e, em alguns casos, superação dos níveis de emissão pré-pandemia evidenciam que pouco se avançou em termos de descarbonização das principais matrizes energéticas durante a crise sanitária, o que representa uma perda de oportunidade quanto aos investimentos de longo prazo em infraestrutura para a mitigação das mudanças climáticas. As negociações realizadas durante a COP-26 em Glasgow, na Escócia, bem como o relatório do IPCC publicado em 2021, demonstram especialmente que os esforços dos governos nacionais não foram suficientes para que a desaceleração econômica causada pela pandemia inaugurasse um ponto de 
virada no enfrentamento à crise climática. Dados recentes atestam a existência de um "efeito rebote", como também a retomada dos investimentos de longo prazo em tecnologias intensivas do ponto de vista do carbono, apontando para um cenário de retrocessos em termos de governança global do clima.

Gráfico 1 - Alterações observadas no início da Pandemia de COVID-19
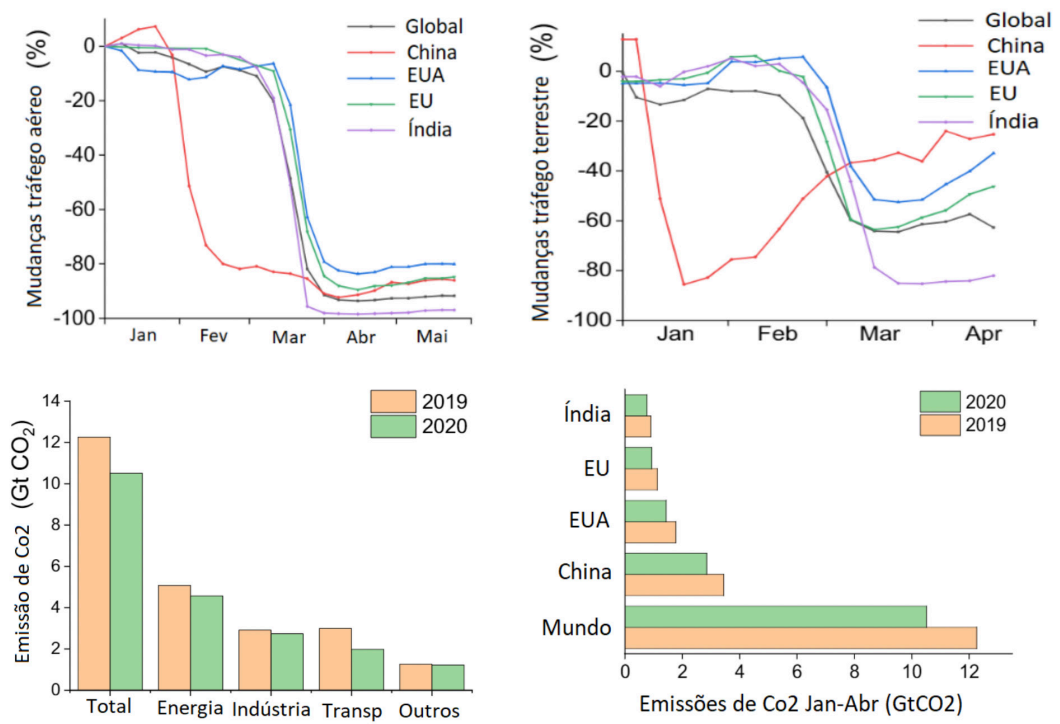

Fonte: Sikarwar et al (2021).

Gráfico 2 - Evolução mensal das emissões globais de CO2 (2020)

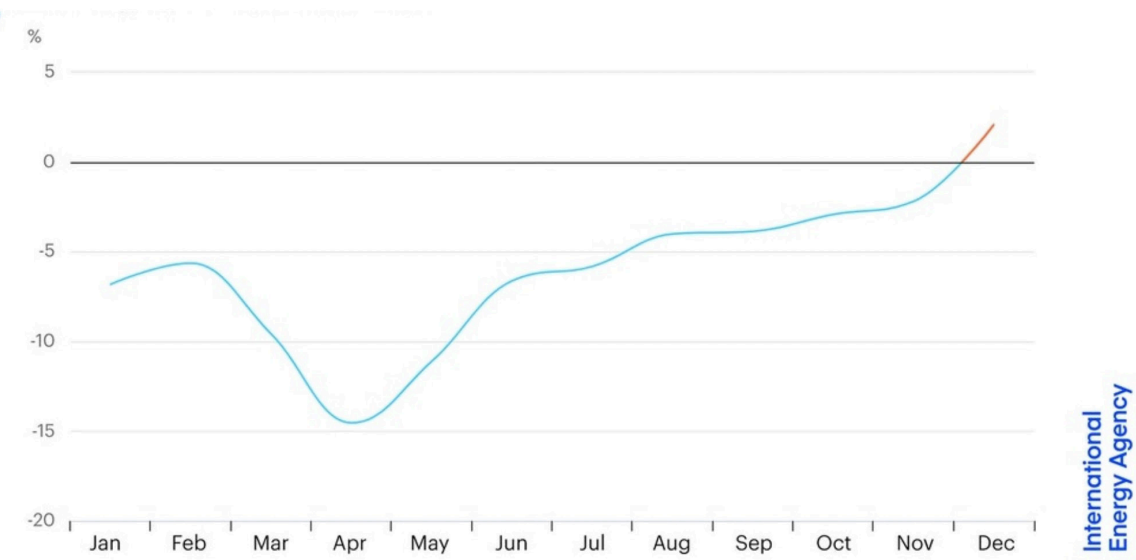

Fonte: IEA (2021). 


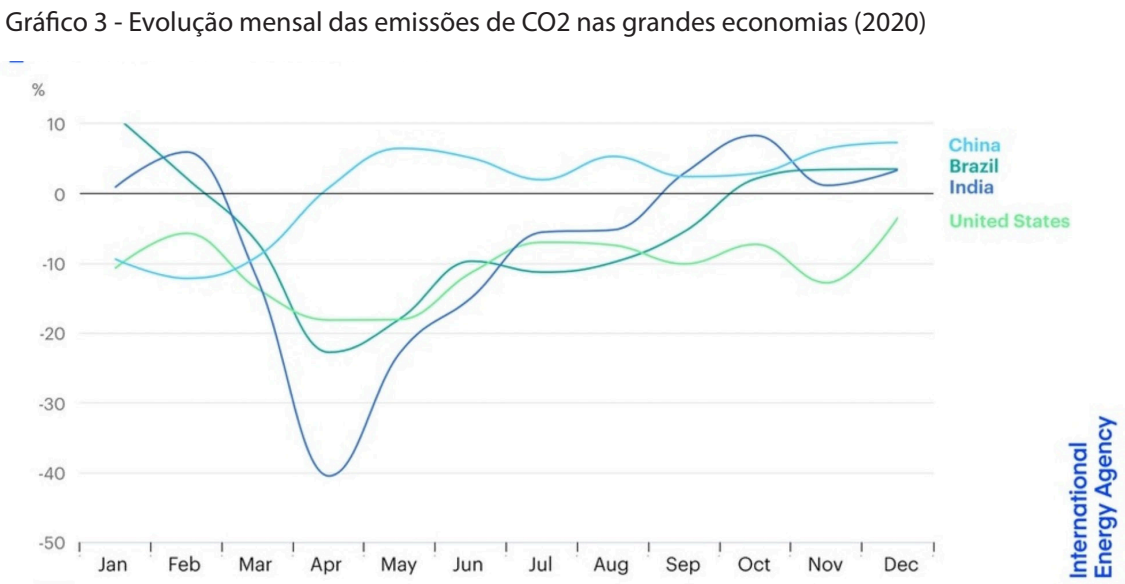

Fonte: IEA (2021).

Apesar dos fortes impactos sanitários e econômicos da pandemia no Brasil, observa-se, de maneira análoga, que o decréscimo de emissões no início de 2020 não se manteve ao longo dos meses seguintes. Pelo contrário, o acumulado no ano representou um acréscimo de 9,5\% com relação a 2019 (Watanabe, 2021). Esse aumento decorre, sobretudo, de "mudanças no uso da terra”, que englobam o desmatamento da Amazônia e do Cerrado. Dados do INPE indicam que, por conta do desmatamento e das alterações no clima, certas regiões da floresta amazônica já emitem mais CO2 do que são capazes de absorver. Essa mudança de padrão no "carbon budget" da floresta é mais nítida em sua porção oriental, onde $30 \%$ da área já se encontra devastada (Qin et al., 2021). A atuação de “milícias ambientais", estimuladas pelo governo Bolsonaro e pelo sistemático desmantelamento de órgãos de fiscalização e controle, apoiou-se na combinação de violência com instrumentos de tecnologia avançada para promover um aumento sem precedentes na devastação desses biomas, transformando o Brasil em pária nas negociações multilaterais sobre o meio ambiente (Souza et al., 2021).

Esses dados têm fomentado debates globais acerca das estratégias de recuperação econômica, aliadas à conservação florestal e à adoção de “energias limpas". Em um contexto de mudanças climáticas com impactos socioambientais severos - ondas de calor e secas mais frequentes e intensas, prejuízos à segurança alimentar das sociedades, perda acelerada de biodiversidade, aumento do nível dos oceanos e potenciais migrações em 
massa -, as metas de redução das emissões estão cada vez mais distantes de serem atingidas (IPCC, 2021). O "efeito rebote" observado ao longo de 2020 representa, em síntese, mais uma oportunidade desperdiçada para que se reconfigurassem os marcos da política climática em nível global.

\section{As implicações da pandemia sobre a governança ambiental global}

A crise de saúde pública instaurada pela pandemia de Covid-19 apresenta uma série de paralelos com as questões relativas ao aquecimento global e pode ser enquadrada como recurso heurístico para adensarmos as reflexões sobre a crise climática. Além de se constituírem fenômenos globais com repercussões regionais específicas, ambas colocam em destaque: (i) a relevância do conhecimento científico e o papel dos experts no debate público; (ii) a importância de se combinar diferentes níveis de medidas de prevenção com respostas de adaptação aos impactos provocados; (iii) a necessidade de intervenção para a proteção das camadas sociais mais vulneráveis; (iv) a ênfase numa cooperação transversal para o enfrentamento dessas crises; (v) o aperfeiçoamento dos instrumentos de comunicação para garantir o engajamento da sociedade civil (Long et al., 2020).

Se levarmos em consideração as repercussões da pandemia sobre as atividades econômicas, contudo, os vínculos entre o surto de Covid-19 e as mudanças climáticas deixam de ser meras analogias para assumirem o plano das relações causais. Conforme argumentamos na seção anterior, as diversas medidas de lockdown e isolamento social provocaram reduções diárias nas emissões de CO2 em virtude de seus impactos sobre o consumo de energia, a produção industrial, a desaceleração do comércio e dos fluxos de transporte terrestre e aéreo (Le Queré et al., 2020). Apesar disso, é evidente que as restrições adotadas tiveram como estratégia fundamental a diminuição das taxas de contágio e que as mudanças na composição da química atmosférica não desempenharam qualquer papel, mesmo que secundário, na formulação dessas diretrizes. Assim, não se trata de abordar essa relação de causalidade como decorrente de uma ação instrumental com finalidades ambientais, mas como subproduto ou consequência não intencionada dos esforços para proteger os cidadãos de uma doença letal. 
Num segundo momento, porém, as relações indiretas entre a pandemia e as mudanças climáticas foram alçadas ao âmbito discursivo de modo a reatualizar conflitos preexistentes à emergência da Covid-19. Por conta de seus impactos negativos na economia global, instituições públicas e agentes de mercado construíram articulações que apresentaram o novo cenário de contração da atividade econômica como fundamento para legitimar a flexibilização das normas ambientais. Nos Estados Unidos, a Agência de Proteção Ambiental (EPA) anunciou que adiaria a entrada em vigor de novas regras de proteção ao meio ambiente e por isso foi criticada por lançar mão do coronavírus como uma "licença para poluir" (Friedman, 2020). Paralelamente, o lobby europeu das montadoras de carros requisitou um alargamento do prazo de adaptação às normas que estabelecem a redução da emissão de CO2 por veículos automotivos (Topham \& Harvey, 2020). Já, no Brasil, a concentração do debate público em torno da pandemia favoreceu os agentes que se beneficiam do desmatamento, não apenas na região Amazônica, mas também em outros biomas, como o Cerrado. Somente na Bahia, 34.307,312 hectares foram desmatados com autorização do governo estadual no período entre a data do decreto de isolamento social (20/03/2020) e o final de junho (AATR, 2020).

A queda nas emissões de poluentes atmosféricos e gases responsáveis pelo efeito estufa, constatada a partir das medidas de lockdown, representou, a princípio, somente um efeito temporário da contração econômica e esteve longe de garantir soluções efetivas para a qualidade do ar nos centros urbanos ou para a emergência climática. Para que as metas do Acordo de Paris possam ser alcançadas, a queda nas emissões de CO2 projetada em 8\% para 2020 deveria se repetir anualmente até atingir um balanço de carbono neutro [netzero emissions] em 2050 (Hepburn et al., 2020). Após atingir seu mínimo em abril, as emissões voltaram a subir e, em dezembro do mesmo ano, já se mostravam 2\% (60 milhões de toneladas) superiores a dezembro de 2019.

O rebote nas emissões de carbono no final do ano passado é um forte aviso de que não se fez o suficiente para acelerar a transição energética mundialmente. (...) Isso pode colocar em risco a oportunidade histórica de fazer com que 2019 representasse o pico definitivo das emissões globais. (IEA, 2021:1)

As circunstâncias advindas da pandemia realçam, portanto, a necessidade de mudanças nas estruturas produtivas, nos parâmetros 
institucionais (nacionais e multilaterais) e debates mais consequentes sobre os padrões de consumo responsáveis pela degradação dos ecossistemas e perda de biodiversidade. Se quisermos evitar que as emissões cresçam vertiginosamente após a crise, é fundamental que os Estados intervenham para que as respostas à emergência climática sejam tão prioritárias quanto a geração de empregos nas diretrizes para a recuperação da economia. Na ausência de um programa que internalize a perspectiva ambiental, o cenário subsequente à Covid-19 pode seguir o mesmo modelo da crise financeira de 2008/2009, quando o impacto sobre os mercados globais resultou numa diminuição de 1,4\% nas emissões de CO2, mas que logo em 2010, foi seguida por um aumento de 5,9\%, impulsionado pelos resultados da indústria de cimento e do consumo de combustíveis fósseis. Em outras palavras, observou-se em 2009 um decréscimo de curtíssimo prazo que teve como "efeito rebote" no ano seguinte o que até então figurava como recorde absoluto em emissões totais e a maior taxa de crescimento nos volumes de CO2 desde 2003 (Peters et al., 2012).

O paralelo com a crise financeira global de 2008/2009 não significaria que a recuperação econômica pós-coronavírus devesse, necessariamente, provocar um novo "efeito rebote" em termos de uma concentração de CO2 na atmosfera. As crises do petróleo de 1973 e 1979, por exemplo, tiveram consequências ambientais relativamente benéficas, porque o aumento nos preços do barril alavancou projetos de eficiência energética e uma parcial substituição desse recurso pelo gás natural. De qualquer forma, mesmo que as consequências da presente crise ainda se encontrem parcialmente em aberto, a conjunção entre pandemia e recessão econômica em alguns países [pancession] nos coloca diante de uma encruzilhada de futuros climáticos. Isso porque os pacotes de recuperação econômica englobam medidas de infraestrutura - energia, transporte, construção civil - que representam decisões de longo prazo em termos de emissões de carbono [carbon lock-in].

Se atentarmos para o exemplo da China, onde as restrições à mobilidade foram relaxadas e as fábricas reabertas antes que em outros países, o "efeito rebote" já pode ser observado. Os estímulos econômicos do governo chinês devem sobrepujar as melhorias ambientais de curto prazo de forma semelhante ao que ocorreu após a desaceleração interna de 2015, visto que, ao longo dos primeiros dezoito dias do mês de março, foram aprovadas centenas de novas usinas termoelétricas movidas a carvão, perfazendo 7.960 MW, mediante um total de 6.310 MW para todo o ano de 2019 (Myllyvirta, 
2020). Em maio de 2021, as emissões chinesas de CO2 já haviam subido 7\% em relação ao período anterior à pandemia - a maior taxa de crescimento desde 2012 -, sendo que 70\% do incremento no primeiro trimestre desse ano se deve ao incremento no uso de carvão. Além disso, a China enfrenta problemas estruturais de excesso de capacidade em setores da indústria considerados intensivos do ponto de vista das emissões de carbono e que podem ser reativados tão logo o mercado global reaqueça a demanda. Mesmo que essa retomada provavelmente também abarque as indústrias de "tecnologias limpas", o plano quinquenal vigente reconhece que suas emissões podem ainda subir mais 5 a 10\% até 2025 (Souza, 2021).

No caso da Europa, dezessete países assinaram uma declaração de intenções para que a resposta à crise econômica decorrente da Covid-19 seja pautada pelos preceitos de um "Green New Deal" que acelere o cumprimento das metas climáticas do Acordo de Paris. Já a Comissão Europeia anunciou o programa Next Generation EU, no qual as "lições da pandemia” se traduziriam em investimentos adicionais de 750 bilhões de euros até 2027, de modo que a saída da crise seja orientada pelo fortalecimento dos sistemas de saúde, expansão das energias renováveis, economia circular, projetos sustentáveis de transporte e logística, além de focar na resiliência socioeconômica de seus países membros (European Comission, 2020). Contudo, mesmo que esses documentos caracterizem a pandemia como um marco para a mobilização contra as mudanças climáticas e a perda de biodiversidade, eles não apontam caminhos que escapem à atual justaposição de projetos de modernização ecológica e atividades que aumentam a pressão sobre as fronteiras ecossistêmicas. Assim, a "face de Janus" da política ambiental europeia se revela, por exemplo, no fato de que, entre março e maio, o Banco Central Europeu injetou 7,6 bilhões de euros em companhias que se dedicam à exploração de combustíveis fósseis (Greenpeace, 2020).

Após a vitória de Biden nas eleições presidenciais de 2020, a proposta de um Green New Deal veio alinhada com a ideia de promover a transição energética e combater tanto a estagnação dos salários quanto a crescente desigualdade social nos Estados Unidos. Diferentemente da modalidade defendida pelo economista Thomas Friedman em 2008, o novo plano demonstrava semelhanças com o programa apresentado por políticos progressistas, como Alexandria Ocasio-Cortez e Bernie Sanders, por enfatizar a coordenação e os investimentos públicos enquanto instrumentos 
para a descarbonização da economia. No entanto, a pressão de congressistas reticentes com as pressões inflacionárias desse programa (inclusive entre membros da bancada Democrata), obrigou o presidente a cortar pela metade os valores inicialmente destinados ao programa. Em que pese seu avanço em relação à política negacionista de Donald Trump, os gastos previstos para o Green New Deal foram reduzidos à metade. Além disso, a promessa de destinar US\$ 555 bilhões para o clima não impediu Biden de, às vésperas da COP 26, conclamar as empresas de gás e petróleo a aumentarem a produção, como corretivo para o aumento no preço da gasolina, nem tampouco de rever os subsídios anuais de US\$ 20 bilhões às empresas de combustíveis fósseis (Tankersley \& Friedman, 2021).

Por outro lado, um grupo de 170 acadêmicos holandeses publicou um manifesto que toma o pulso de decrescimento não intencionado resultante dos lockdowns como base para a construção de uma agenda de decrescimento econômico. Isso não quer dizer que as medidas de isolamento social sejam por eles encaradas como instrumentos para a mitigação do aquecimento global, mas reconhecem que nenhum plano de recuperação econômica baseado nos princípios de crescimento ilimitado pode superar a presente crise sem nos lançar em outra ainda mais catastrófica. Tal manifesto apresenta um plano de ação fundamentado em cinco propostas: (i) superação do imperativo de crescimento agregado do PIB - que se fundamentaria na diferenciação entre o fomento aos serviços públicos e o desincentivo àquelas atividades fundamentalmente insustentáveis como os setores petrolífero, mineração, publicidade e consumo de luxo; (ii) construção de um modelo econômico pautado pela distribuição de riquezas e orientado por sistemas fiscais de taxação progressiva e mecanismos de renda mínima; (iii) agricultura regenerativa baseada no locavorismo e vegetarianismo para a preservação da biodiversidade; (iv) redução do consumo de luxo, das viagens de longa distância e do desperdício; (v) cancelamento das dívidas de trabalhadores, pequenos empresários e dos países do Sul Global (Feola, 2020).

Num sentido análogo às propostas de decrescimento, a municipalidade de Amsterdã aprovou, em abril do ano passado, a implementação de medidas de recuperação pautadas pelo "modelo Donut", desenvolvido por pesquisadores da Universidade de Oxford, segundo o qual as metas de desenvolvimento humano são, por definição, indissociáveis de políticas públicas que levem em consideração os limites planetários (Boffey, 2020). 
No entanto, conforme argumentaremos na próxima seção, o problema decisivo vem a ser como os instrumentos democráticos e de cooperação, em múltiplos níveis de governança, poderiam permitir a superação dos presentes obstáculos, de modo a que os esforços para se conjugar recuperação econômica e sustentabilidade não se resumam a meros artifícios retóricos ou experiências isoladas.

\section{Os limites da ciência e da democracia na prevenção de catástrofes anunciadas}

"Não desperdicem esta crise”, concluiu o prêmio Nobel de economia Joseph Stiglitz durante entrevista sobre a pandemia de Covid-19 (Stiglitz, 2020). O alerta de Stiglitz diz respeito ao contexto atual, mas também expressa a percepção de que o século 20 nos deixou uma coleção de crises desperdiçadas. Com efeito, a tendência de elevação das temperaturas médias já havia sido constatada por Svante August Arrhenius em 1896, quando o químico sueco associou o aumento da concentração de dióxido de carbono na atmosfera à queima de combustíveis fósseis. Foi somente na década de 1970, no entanto, que as mudanças climáticas foram reconhecidas como ameaças de caráter antropogênico que poderiam colocar em xeque a existência das civilizações humanas tal como as conhecemos (Bolin, 2007). Esse acúmulo de conhecimento perito teve uma série de capítulos importantes, como a fundação, nos Estados Unidos, da Union of Concerned Scientists (1969), que se propunha a tarefa de enfrentar problemas ambientais e sociais para os quais apontavam os estudos científicos mais recentes (Edgerton, 2006; Moore, 2009). No ano anterior, a criação do Clube de Roma também representou um marco político na história das ciências ambientais que, a partir de então, tornariam mais explícitos em seus prognósticos a incompatibilidade entre os padrões de desenvolvimento das sociedades industriais e o equilíbrio ecossistêmico (Caradonna, 2014).

É nesse contexto que o relatório sobre Os limites do crescimento apontou os limites de absorção da biosfera quanto ao ritmo exponencial do aumento populacional, da produção industrial e de alimentos, de seus rejeitos poluentes e do crescimento econômico em geral (Meadows et al., 1972). De acordo com Paul Edwards (2010), o aporte de Meadows e seus colaboradores 
havia sido um dos primeiros a utilizar de maneira bem-sucedida a simulação computacional de cenários futuros como método de investigação. O relatório chamou a atenção de vários agentes e instituições (Simmons, 2000; Mackenzie, 2012), sendo que novas simulações, realizadas 40 anos depois e com recursos de pesquisa mais sofisticados, viriam a corroborá-lo em seus principais aspectos. A acurácia desses prognósticos não garantiu, todavia, que medidas práticas fossem implementadas para evitar cenários distópicos (Turner \& Alexander, 2014).

De maneira análoga aos estudos de modelagem ambiental que anteciparam as consequências das mudanças climáticas e da ultrapassagem de outras fronteiras ecossistêmicas, o nexo causal entre desmatamento e novas epidemias já vinha sendo apontado como fator de risco para crises sanitárias como a instalada pela Covid-19. Há pelo menos duas décadas, os cientistas repetem o alerta de que o avanço sobre as florestas aumenta a probabilidade de que as populações humanas entrem em contato com microrganismos desconhecidos, seja diretamente ou por intermédio de espécies hospedeiras, como morcegos, gado bovino, suíno e aves. Ecólogos e epidemiologistas também assinalam que o desmatamento acelerado da Amazônia e de regiões da África e do Sudeste Asiático pode convertê-las em focos irradiadores de epidemias no futuro próximo (Pontes, 2020). As mudanças climáticas e no uso da terra, juntamente com seus impactos sobre a biodiversidade, exacerbariam as interações entre humanos e microrganismos desconhecidos de forma a potencializar o surgimento de novas formas de contágio, sendo que quadros dirigentes da OMS já afirmam que "uma nova pandemia é inevitável" (Carlson et al., 2021).

Nesse sentido, podemos afirmar que o "paradoxo de Giddens" expressa um drama existencial que diz respeito tanto às mudanças climáticas quanto aos prognósticos de novas pandemias. Tais quais os perigos do aquecimento global, os riscos de contágio por vírus ainda desconhecidos representam ameaças não palpáveis, imediatas ou visíveis no decorrer da vida cotidiana. Mesmo que embasados por investigações científicas criteriosas, esses riscos assumem um caráter abstrato e recebem atenção insuficiente por parte das elites políticas e da sociedade civil porque são vistos como cenários distantes, aos quais não se atribui o mesmo nível de realidade que às preocupações do presente. Como as ações preventivas são sistematicamente adiadas, entretanto, tais riscos deixam de figurar como distopias longínquas 
para darem lugar a crises que desorganizam o conjunto das sociedades. Dessa forma, a opção por desviar o olhar redunda em colapsos tão tangíveis e agudos que, ou as ações corretivas se tornam ainda mais custosas, ou já será tarde demais para quaisquer medidas capazes de reverter a catástrofe (Giddens, 2010).

Com a ascensão do neoliberalismo, as forças de mercado viram-se paulatinamente desembaraçadas não apenas das garantias trabalhistas e da seguridade social que balizaram o welfare state, como de constrangimentos legais para a exploração desenfreada dos recursos naturais - particularmente naquelas nações do Sul global que se enquadram na divisão internacional do trabalho como provedoras de commodities. No entanto, a degradação acelerada do meio ambiente - seja pela flexibilização de marcos legais de conservação, seja pela ação rapineira das "ecomáfias" (Souza et al., 2021; Unep/Interpol, 2016) - assume paradoxalmente a forma de uma colisão suicida do modelo neoliberal, uma vez que a relação entre economia e ecossistemas assume contornos cada vez mais disfuncionais. Conforme argumenta Luiz Marques, "não é mais plausível esperar, passada a pandemia, um novo ciclo de crescimento econômico global e ainda menos nacional. Se algum crescimento voltar a ocorrer, ele será conjuntural e logo truncado pelo caos climático, ecológico e sanitário” (Marques, 2020a, p. 14).

Ainda segundo Luiz Marques (2020b), as sinergias entre as crises econômica, ambiental e sanitária colocariam na ordem do dia uma agenda política fundamentalmente defensiva e adaptativa que deve gravitar em torno de um programa voltado para a sobrevivência das sociedades. A construção desse programa se realizaria num cenário de maiores desigualdades, disfunções econômicas, empobrecimento biológico e poluição generalizada, razões pelas quais essa disputa representaria uma plataforma política muito mais ambiciosa que os programas socialdemocratas ou revolucionários do século 20. Isso implicaria uma guinada histórica que tornaria obsoletos aqueles programas ambientalistas que se orientam pela mera justaposição de critérios de sustentabilidade a um modelo econômico estruturalmente insustentável e incapaz de resolver os problemas por ele mesmo engendrados. Em outras palavras, significaria uma redefinição do próprio sentido da atividade econômica e do nosso lugar enquanto espécie no interior da biosfera.

Em que pesem as contradições apontadas na seção anterior, Mastini e colaboradores (2021) argumentam que os programas da União Europeia 
e dos Estados Unidos para fomentar o Green New Deal ainda figurariam como os mais promissores esboços de legislação social e ambiental, se efetivamente atrelados à criação de empregos em setores voltados para a descarbonização da economia e comprometidos com os movimentos por justiça ambiental nos países do Sul global. Eles reconhecem, porém, a validade da premissa dos teóricos do decrescimento de que os estímulos aos investimentos nos setores da "economia limpa" trazem consigo consequências indesejáveis de segunda ordem, tais como a expansão de setores poluentes. É claro que algumas categorias da atividade econômica deveriam crescer massivamente no contexto da transição ecológica, mas não se deve esquecer que o crescimento é um processo integrado que não permite por mera força do desejo incrementar seletivamente os setores "verdes" sem que haja também algum aumento na demanda por produtos que implicam maior poluição. Por isso esses autores sugerem que o Green New Deal deve ser considerado uma estratégia de transição, cujas reformas apontariam no longo prazo para a plataforma política do decrescimento (Mastini et al., 2021).

Por mais sedutora que pareça essa formulação, é difícil imaginar como o Green New Deal poderia mesmo figurar como estratégia de transição para o decrescimento no atual contexto de acirramento de disputas geopolíticas e da incessante corrida armamentista entre as grandes potências (EUA, China, Rússia e UE). Somente nas duas últimas décadas, os Estados Unidos gastaram 6,4 trilhões de dólares em guerra, ou seja, um valor 42\% superior aos 4,5 trilhões que seriam necessários para realizar a transição de sua matriz energética para fontes cem por cento renováveis. Com efeito, a expansão dos aparatos militares demanda crescimento econômico e controle das fontes de combustíveis fósseis. Militarismo e crise climática estão inter-relacionados e se reforçam mutuamente. O setor militar figura entre os mais poluentes e é frequentemente empregado para sustentar indústrias extrativistas que desestabilizam o clima. O caos climático, por sua vez, leva a deslocamentos populacionais em massa, à militarização das fronteiras e à perspectiva de novos conflitos (Steichen \& Koshgarian, 2021). O caso dos EUA é, de fato, emblemático. Para atualizar os dados de Paulo Artaxo (2020), o país gasta anualmente trilhões de dólares em armas nucleares e outras máquinas de guerra. Mas um simples vírus de 120nm causou a morte de 780 mil norteamericanos em menos de dois anos. 


\section{Referências}

Artaxo, Paulo. (2020). As três emergências que nossa sociedade enfrenta: saúde, biodiversidade e mudanças climáticas. Estudos Avançados, 34(100), 53-66. https://doi.org/10.1590/s0103-4014.2020.34100.005

Associação dos Advogados dos Trabalhadores Rurais (AATR). (2020, 1 jul.). Bahia já autorizou o desmatamento de 34.307,712 ha durante a pandemia. AATR. https://www.aatr.org.br/post/bahia-j\%C3\%A1autorizou-o-desmatamento-de-34-307-712-ha-durante-a-pandemia

Boffey, Daniel. (2020, 8 abr.). Amsterdam to embrace "doughnut" model to mend post-coronavirus economy. The Guardian. https://www. theguardian.com/world/2020/apr/08/amsterdam-doughnut-modelmend-post-coronavirus-economy

Bolin, Bert. (2007). A history of the science and politics of climate change: the role of the Intergovernmental Panel on Climate Change. Cambridge University Press.

Caradonna, Jeremy. (2014). Sustainability: a history. Oxford University Press.

Calrson, Colin J., Albery, Gregory F., \& Phelan, Alexandra. (2021). Preparing International Cooperation on Pandemic Prevention for the Anthropocene. BMJ Global Health, 6(3), e004254. https://doi. org/101136/bmjgh-2020-004254

Chen, Kai, Wang, Meng, \& Huang, Conghong. (2020). Air pollution reduction and mortality benefit during the COVID-19 outbreack in China. The Lancet Health, 4(6), e210-e212. https://doi.org/10.1016/s2542$\underline{5196(20) 30107-8}$

Chinazzi, Matteo, Davis Jessica, \& Ajelli, Marco. (2020). The effect of travel restrictions on the spread of the 2019 novel coronavirus (COVID-19) outbreak. Science, 368(6489), 395-400. https://doi.org/10.1126/ $\underline{\text { science.aba9757 }}$

Chintalapudi, Nalini, Battineme, Gopi, \& Amenta, Francesco. (2020). COVID-19 virus outbreak forecasting of registered and recovered cases after sixty-day lockdown in Italy: A data driven model approach. Journal of Microbiology, Immunology and Infection, 53(3), 396-403. https://doi.org/10.1016/j.jmii.2020.04.004

Cucinotta, Domenico, \& Vanelli, Maurizio. (2020). WHO Declares COVID-19 a Pandemic. Acta Bio-medica: Atenei Parmensis, 91(1), 157-160. https://doi.org/10.23750/abm.v91i1.9397 
Dantas, Guilherme, Siciliano, Bruno, França, Bruno B., Silva, Cleyton M., \& Arbilla, Graciela. (2020). The impact of COVID-19 partial lockdown on the air quality of the city of Rio de Janeiro, Brazil. Science of Total Environment, (729), 139085. https://dx.doi.org/10.1016\%2Fj. scitotenv.2020.139085

Dolhnikoff, Marisa et al. (2020). Pathological evidence of pulmonary thrombotic phenomena in severe COVID-19. Journal of Thrombosis and Haemostasis, 18(6), 1517-1519. https://doi.org/10.1111/jth.14844

Edgerton, David. (2006). Warfare State: Britain, 1920-1970. Cambridge University Press.

Edwards, Paul N. (2010). A vast machine: computer models, climate data, and the politics of global. The MIT Press.

European Comission. (2020). Europe's moment: repair and prepare for the next generation. European Comission. https://ec.europa.eu/ commission/presscorner/detail/en/ip_20_940

Feola, Giuseppe. (2020, 16 abr.). Manifesto for post-neoliberal development: five policy strategies for the Netherlands after the Covid-19 crisis. Ontgroei.https://ontgroei.degrowth.net/manifesto-for-post-neoliberaldevelopment-five-policy-strategies-for-the-netherlands-after-thecovid-19-crisis/

Friedman, Lisa. (2020, 10 jun.). E.P.A, citing Coronavirus, drastically relaxes rules for polluters. The New York Times. https://www.nytimes. com/2020/03/26/climate/epa-coronavirus-pollution-rules.html

GERIMA - Grupo de Pesquisa em Relações Internacionais e Meio Ambiente. (2020, 17 abr.). Covid-19 e a emergência climática: conexões e desafios. Le Monde Diplomatique. https://diplomatique.org.br/covid-19-e-aemergencia-climatica-conexoes-e-desafios/

Greenpeace. (2020). ECB injects over $€ 7$ billion into fossil fuels since start of COVID-19 crisis. Greenpeace. https://www.greenpeace.org/eu-unit/ issues/climate-energy/3933/ecb-injects-e7-billion-into-fossil-fuelscoronavirus-crisis/

Giddens, Anthony. (2010). A política da mudança climática. Zahar.

Gilbert, Dominic. (2020, 19 abr.). Which countries are under lockdown - and is it working?. The Telegraph. https://www.telegraph.co.uk/ news/2020/04/16/countries-in-lockdown-denmark-germany/

Harvey, Fiona. (2020, 11 dez.). Rebound in carbon emissions expected in 2021 after fall caused by Covid. The Guardian. https://www. theguardian.com/environment/2020/dec/11/rebound-in-carbonemissions-expected-in-2021-after-fall-caused-by-covid 
Hepburn, Cameron, O’Callaghan, Brian, Stern, Nicholas, Stiglitz, Joseph, \& Zenghelis, Dimitri. (2020). Will COVID-19 fiscal recovery packages accelerate or retard progresso $\mathrm{n}$ climate change? Oxford Review of Economic Policy, 36(S1), S359-S381. http://doi.org/10.1093/oxrep/graa015

IEA - International Energy Agency. (2021). After Steep drop in Early 2020, Global Carbon Emissions have Rebounded Strongly. IEA. https://www. iea.org/news/after-steep-drop-in-early-2020-global-carbon-dioxideemissions-have-rebounded-strongly

IPCC - Intergovernmental Panel on Climate Change. (2021). AR6 climate change 2021: the physical science basis. IPCC. https://www.ipcc.ch/ report/ar6/wg1/

Kerimray, Aiymgul, Baimatova, Nassiba, Ibragimova, Olga P., Bukenov, Bauyrzhan, Kenessov, Bulat, Plotitsyn, Pavel, \& Karaca, Ferhat. (2020). Assessing air quality changes in large cities during COVID-19 lockdowns: the impacts of traffic-free urban conditions in Almaty, Kazakhstan. Science of the Total Environment, (730), 139179. https:// doi.org/10.1016/i.scitotenv.2020.139179

Krecl, Patricia, Targiro, Admir, Oukawa, Gabriel Y., \& Cassino Jr., Regis P. (2020). Drop in air pollution from COVID-19 pandemic: policy implications for the megacity of São Paulo. Environment Pollution, (265), 114883. https://doi.org/10.1016/j.envpol.2020.114883

Kucharski, Adam J. et al. (2020). Early dynamics of transmission and control of COVID-19: a mathematical modelling study. The Lancet: Infectious Diseases, 20(5), 553-558. https://doi.org/10.1016/S14733099(20)30144-4

Lal, Preet et al. (2020). The dark cloud with a silver lining: assessing the impact of SARS COVID-19 pandemic on the global environment. Science of the Total Environment, (732), 139297. https://doi. org/10.1016/j.scitotenv.2020.139297

Le-Queré, Corinne, Jackson, Robert B., \& Jones, Matthew W. (2020). Temporary reductions in daily global CO2 emissions during the COVID-19 forced confinement. Nature Climate Change, 10, 647-653. https://doi.org/10.1038/s41558-020-0797-x

Li, Xinhai, Zhao, Xumao, \& Sun, Yuehua. (2020). “The lockdown of Hubei Province causing different transmission dynamics of the novel coronavirus (2019-nCoV) in Wuhan and Beijing”. MedRxiv, preprint. https://doi.org/10.1101/2020.02.09.20021477

Long, Jessica, Gordon, Lizzie, \& Townend, Ruth. (2020). Now what? Climate change $\&$ Coronavirus. Ipsos. 
Mackenzie, Debora. (2012, 4 jan.). Boom and doom: revisiting prophecies of collapse. New Scientist. https://www.newscientist.com/article/ mg21328462-100-boom-and-doom-revisiting-prophecies-of-collapse/

Marques, Luiz. (2020a, 5 maio). A pandemia incide no ano mais importante da história da humanidade. Serão as próximas zoonoses gestadas na Amazônia? Jornal da Unicamp. https://www.uni-camp.br/unicamp// index.php/noticias/2020/05/05/pandemia-incide-o-ano-maisimportante-da-historia-da-humanidade-serao-proximas

Marques, Luiz. (2020b). Pandemics, existential and non-existential risks to humanity. Ambiente \& Sociedade, (23), 1-9. https://doi. org/10.1590/1809-4422asoc20200126vu2020L3ID

Mastini, Riccardo, Kallis, Giorgio, \& Hackel, Jason. (2021). A Green New Deal without growth. Ecological Economics, 179(C), 1-9. https://doi. org/10.1016/j.ecolecon.2020.106832

Mcneill, Faye V. (2020). COVID-19 and the air we breathe. Earth and space chemistry, 4(5), 674-675. https://dx.doi.org/10.1021/ acsearthspacechem.0c00093

Meadows, Donella H, Meadows, Dennis L, Randers, Jørgen, Behrens III, William W. (1972). The limits to growth: a report for the club of Rome's project on the predicament of mankind. Universe Books.

Moore, Mark. (2009). The Union of Concerned Scientists on the uncertainty of climate change: a study of synecdochic form. Environmental Communication, 3(2), 191-205.

Morens, David, Daszak, Peter, Markel, Howard, \& Taubenberger, Jeffery K. (2020). Pandemic COVID-19 joins History's pandemic legion. MBio, 11(3), e00812-20. https://doi.org/10.1128/mBio.00812-20

Myllyvirta, Lauri. (2020, 19 fev.). Analysis: Coronavirus temporarily reduced China's CO2 emissions by a quarter. CarbonBrief. https:// www.carbonbrief.org/analysis-coronavirus-has-temporarily-reducedchinas-co2-emissions-by-a-quarter

Nakada, Liane, \& Urban, Rodrigo. (2020). COVID-19 pandemic: Impacts on the air quality during the partial lockdown in São Paulo state, Brazil. Science of the Total Environment, (730), 139087. https://doi. org/10.1016/j.scitotenv.2020.139087

Peters, Glen P., Marland, Gregg, Le Quéré, Corinne, Boden, Thomas, Canadell, Josep G., \& Raupach, Michael R. (2012). Rapid growth in CO2 emissions after the 2008-2009 global financial crisis. Nature Climate Change, (2), 2-4. https://doi.org/10.1038/nclimate1332 
Pontes, Nadia. (2020, 15 abr.). O elo entre desmatamento e epidemias investigado pela ciência. Deutsche Welle. https://www.dw.com/ pt-br/o-elo-entre-desmatamento-e-epidemias-investigado-pelaci\%C3\%AAncia/a-53135352

Qin, Yuanwei et al. (2021). Carbon loss from forest degradation exceeds that from deforestation in the Brazilian Amazon. Nature Climate Change, (11), 442-8. https://doi.org/10.1038/s41558-021-01026-5

Rosenbloom, Daniel, \& Markard, Jochen. (2021). Editorial: A COVID-19 recovery for climate. Science, 368(6490), 447. https://doi.org/10.1126/ $\underline{\text { science.abc } 4887}$

Ren, Xuefei. (2020). Pandemic and lockdown: a territorial approach to COVID-19 in China, Italy and the United States. Eurasian Geography and Economics, 61(4-5), 423-434. https://doi.org/10.1080/15387216.2020.1762103

Shrestha, Asheshwor, Shrestha, Uttam, \& Sharma, Roshan. (2020). Lockdown caused by COVID-19 pandemic reduces air pollution in cities worldwide. EarthArXiv, preprint. https://doi.org/10.31223/osf.io/edt4j

Siciliano, Bruno, Dantas, Guilherme, Silva, Cleyton M., Arbilla, Graciela. (2020). Increased ozone levels during the COVID-19 lockdown: analysis for the city of Rio de Janeiro, Brazil. Science of the Total Environment, (737), 139765. https://doi.org/10.1016/j.scitotenv.2020.139765

Simmons, Matthew. (2000, out.). Revisiting “The Limits to Growth": could the Club of Rome have been correct, after all? Mudcity Pree [An energy white paper]. https://mudcitypress.com/PDF/clubofrome.pdf

Sikarwar, Vineet S., Reichert, Annika, Jeremias, Michal, \& Manovic, Vasilije. (2021). COVID-19 pandemic and global carbon dioxide emissions: a first assessment. Science of the Total Environment, 794, 148770. https://10.1016/j.scitotenv.2021.148770

Souza, Luiz E. V. (2021). Civilização ecológica ou colapso ambiental. In R. Musse (Org.), China contemporânea: seis interpretações (pp. 163-192). Autêntica.

Souza, Luiz. E. V., Fetz, Marcelo, Zagatto, Bruna P., \& Pinho, Nataly S. (2021). Violence and illegal deforestation: the crimes of "environmental militias" in the Amazon Forest. Capitalism Nature Socialism. https:// doi.org/10.1080/10455752.2021.1980817

Steichen, Lorah, \& Koshgarian, Lindsay. (2021). No warming, no militarism: how militarism fuels the climate crisis - and vice versa. Institute for Policy Studies. https://ips-dc.org/climate-militarism-primer/ 
Stiglitz, Joseph. (2020, 5 maio). “Não desperdicem esta crise”. Entrevista de Gianrico Carofiglio com Joseph Stiglitz. Tradução de Luisa Rabolini. Revista IHU, (546). https://www.ihu.unisinos.br/598577-naodesperdicem-esta-crise-entrevista-com-joseph-stiglitz

The Lancet. (2020). India under COVID-19 lockdown. The Lancet, 395(10233), 1315. https://10.1016/S0140-6736(20)30938-7

Tankersley, Jim, \& Friedman, Lisa. (2021, 4 nov.). Even as Biden pushes clean energy, he seeks more oil production. The New York Times. https:// www.nytimes.com/2021/11/01/climate/biden-oil-gas-cop26.html

Topham, Gwyn, \& Harvey, Fiona. (2020, 27 mar.). Carmakers accused of trying to use crisis to avert emissions crackdown. The Guardian. https://www. theguardian.com/business/2020/mar/27/carmakers-accused-of-usingcovid-19-weaken-environmental-laws

Turner, Graham, \& Alexander, Cathy. (2014, 2 set.). "Limits to Growth” was right. New research shows we're nearing collapse. The Guardian. https:/www.theguardian.com/commentisfree/2014/sep/02/limits-togrowth-was-right-new-research-shows-were-nearing-collapse

UNEP/INTERPOL. (2016). The rise of environmental crime. A growing threat to natural resources, peace, development and security [Report].

Wang, Pengfei, Chen, Kaiyu, \& Zhu, Shenqiang. (2020). Severe air pollution not avoided by reduced anthropogenic activities during COVID-19 outbreak. Resources, Conservation and Recycling, 158, 104814. https:// doi.org/10.1016/j.resconrec.2020.104814

Xiao Yonghong, \& Torok, Mili E. (2020). Taking the right measures to control COVID-19. The Lancet: Infectious Diseases, 20(5), 523-524. https://doi. org/10.1016/s1473-3099(20)30152-3

Watanabe, Phillippe. (2021, 28 out.). Brasil aumenta 9,5\% emissões de gases-estufa em 2020, apesar da pandemia. Folha de São Paulo. https://www1.folha.uol.com.br/ambiente/2021/10/brasil-aumenta-95emissoes-de-gases-estufa-em-2020-apesar-da-pandemia.shtml

Zambrano-Monserrate, Manuel, Ruano, Maria A., \& Sanchez-Alcalde, Luis. (2020). Indirect effects of COVID-19 on the environment. Science of the Total Environment, (728), 138813. https://doi.org/10.1016/j. scitotenv.2020.138813

Zhang, Weituo, Qian, Bi-yun. (2020). Making decisions to mitigate COVID-19 with limited knowledge. The Lancet Infectious Diseases, 20(10), 11211122. https://doi.org/10.1016/S1473-3099(20)30280-2 
Zhu, Na, Zhang, Dingyu, \& Wang, Wenling. (2020). A novel coronavirus from patients with pneumonia in China, 2019. New England Journal of Medicine. https://doi.org/10.1056/NEJMoa2001017

\section{(cc) $\mathrm{EY}$}

Licenciado sob uma Licença Creative Commons Attribution 4.0 